\title{
12 Connected Smart Appliances
}

Elgar Fleisch, Hubert Österle und Frederic Thiesse

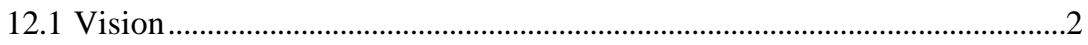

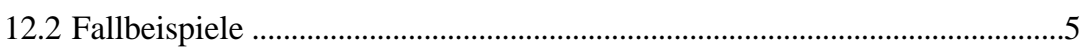

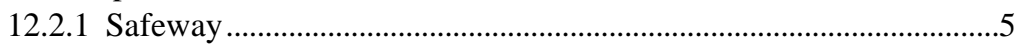

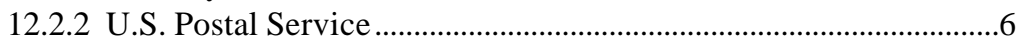

12.2.3 Siemens HomeAssistant....................................................................6

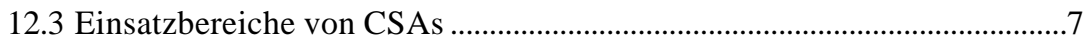

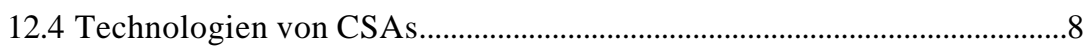

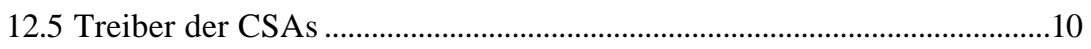

12.6 Betriebswirtschaftliche Effekte von CSAs ................................................13

12.6.1 Neue Prozesse durch Automatisierung der

Datenerfassung ...................................................................................13

12.6.2 Management von komplexen Systemen durch Verteilung von Wissen und Intelligenz ............................................15

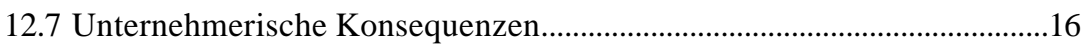

Auszug aus

Österle, H., Fleisch, E., Alt, R., Business Networking in der Praxis, Springer, Berlin 2001 


\subsection{Vision}

Die Entwicklung von Computerhardware war in den letzten Jahrzehnten von einer dramatisch steigenden Rechenleistung auf kleinstem Raum bei rapide sinkenden Kosten geprägt. Dies und die Verfügbarkeit von Multimedia- und InternetTechnologien hat die enorme Popularisierung von Informationstechnologie über den kommerziellen Einsatz hinaus ermöglicht. Die durch den Eintritt in den Massenmarkt erzielten Stückzahlen haben zusammen mit den extrem kurzen Produktlebenszyklen zu nahezu vernachlässigbaren Kosten für leistungsfähige CPU- und Speicherchips geführt. War in der Vergangenheit Digitaltechnik bereits in anderen Hightech-Produkten zu finden (z.B. Waschmaschinen, Automotoren), so ermöglicht der massive Preisverfall nun auch die Integration mit nahezu beliebigen Betriebsmitteln bzw. Gebrauchsgegenständen.

Die Innovation durch IT steht damit erst am Anfang. Sie löst in wenigen Jahren eine weitere Welle neuer Leistungen, Prozesse und Unternehmen aus. Jeder Gegenstand eines Unternehmens kann zu einem Knoten in einem IT-gestützten Netzwerk werden. Beispielsweise erhalten Holzpaletten einen Chip und können dadurch mit anderen physischen Objekten wie z.B. Lagerplätzen, LKWs, Produktionsmaschinen oder Logistiksystemen ohne menschliche Intervention kommunizieren. IT ermöglicht damit eine zeitnahe und detailgetreue Abbildung der betriebswirtschaftlichen Realität und führt zu neuen, heute kaum vorhersehbaren Lösungsansätzen.

Forschung und Praxis konzentrieren sich bis heute auf die Vernetzung von Unternehmen, Prozessen, Informationssystemen und Menschen. Die Diskussion der direkten Vernetzung von Betriebsmitteln bzw. Gebrauchsgegenständen erscheint demgegenüber unterentwickelt.

Die Konvergenz von Informations- und Kommunikationstechnologie (IuK) und Betriebsmitteln bzw. Gebrauchsgegenständen wie Gebäude, Maschinen, Fahrzeuge, Transporteinrichtungen, Einrichtungsgegenstände, Telefon, Mikrowelle, Vdeokamera, Kühlschrank usw. führt zu sogenannten 'Connected Smart Appliances (CSAs)', d.h. Produkten, die Rechenkapazitäten eines PCs besitzen und damit die Funktionalität eines der letztgenannten Geräte erweitern. Mögliche Anwendungsgebiete sind mobile, vernetzte Büros, Haushaltsgeräte mit integriertem Internetanschluß, verschiedenste Informations- und Unterhaltungsangebote die über den Fernseher zugänglich werden, usw.

Die Breite der möglichen Anwendungsgebiete zeigt sich derzeit u.a. auch in der resultierenden Begriffsvielfalt. Einige der hier anzutreffenden Stichworte sind: 
- Embedded Systems

- Home Automation

- Home Networking

- Information Appliances

- Internet Appliances

- Mobile Commerce

- Pervasive Computing

- Smart Appliances

- Ubiquitious Computing

- Wearable Computing

- Wireless Devices

CSAs sind mehr als nur eine neue Generation leistungsfähigerer Taschenrechner und unterscheiden sich von diesen insbesondere durch folgende Eigenschaften (vgl. [Kuri 1999], [Norman 1998, S. 53-57]):

- Connected

CSAs arbeiten nicht von ihrer Umwelt isoliert, sondern verfügen über die Möglichkeit zur Vernetzung. Einerseits können CSAs untereinander Daten austauschen und Funktionalitäten nutzen wie z.B. bei der Verknüpfung einer digitalen Kamera und eines Mobiltelefons zur Übertragung von Fotos vom Urlaubsort aus. Andererseits besteht die Möglichkeit zur Anbindung an externe Dienstleistungen wie z.B. bei der Fernwartung von Haushaltsgeräten über das Internet. Bei fest installierten CSAs kann diese Vernetzung über Telefonoder Stromleitungen realisiert werden; von weitaus größerer Bedeutung ist in diesem Zusammenhang aber die rapid zunehmende Vernetzung mittels drahtloser Datenübertragung.

- $\quad$ Smart

Obwohl CSAs über hohe Rechenkapazitäten verfügen, die bis an die eines PCs heranreichen, wird diese vor dem Benutzer verborgen und genutzt, um das Gerät auf maximale Benutzerfreundlichkeit auszurichten. Während ein PC eine - nach entsprechender Einarbeitungszeit - universell einsetzbare Rechenmaschine darstellt, sind CSAs auf einige wenige Funktionalitäten beschränkt, die dem Benutzer aber mit größtmöglicher Einfachheit und größtmöglichem Komfort zur Verfügung gestellt werden.

- Appliances

Das Navigationssystem des Autos (GPS), das Mobiltelefon (GSM), der Pager, die TV-Set-top-Box, Roadpricing-Systeme, internetfähige Wartungscomputer an Maschinen, das elektronische Buch und elektronische Fußfesseln im Straf- 
vollzug sind Beispiele für vernetzte Geräte, die bereits weitverbreitet im Einsatz sind und einfache Betriebsmittel zu intelligenten, vernetzten Betriebsmitteln verwandeln. Videokameras, Fotoapparate sowie Audio- und Videoanlagen sind zunehmend digitalisiert und haben ebenfalls - zum Teil via PC Anschluß zum Internet gefunden. Eine besondere Form intelligenter Geräte sind Smart Cards in vielfältigen Anwendungsformen, vom Autorisierungsmittel bis zur elektronischen Geldbörse. All diese Geräte ermöglichen zu Hause und unterwegs einen auf das jeweilige Gerät angepaßten Zugriff auf das Internet bzw. Netze einzelner Unternehmen.

CSAs im Sinne der Connected Smart Assets ${ }^{1}$ sind Betriebsmittel bzw. Gegenstände des täglichen Gebrauchs, z.B. Autos, Waschmaschinen, Kleidungsstücke oder Verpackungsmaterial. Es sind sog. 'hybride Produkte', da sie physische Produkte und informationsverarbeitende Elemente in sich vereinigen. Im Extremfall werden CSAs sogar zu einem Bestandteil des menschlichen Körpers, z.B. zur permanenten Fernüberwachung von Herzschlag oder Blutdruck von Patienten in deren eigener Wohnung.

Unter Verwendung von CSAs könnte der Flug eines Geschäftsreisenden in Zukunft wie folgt aussehen: Der Passagier reserviert via Internet oder Call Center seinen Flug. Ein paar Stunden vor dem Abflug erinnert ihn die elektronische Agenda seines Handys an die Bestätigung des Fluges und leitet diese bei Einverständnis des Passagiers an die Fluggesellschaft weiter. Im Falle einer Verspätung sendet die Fluggesellschaft dem Passagier eine SMS-Nachricht oder eine Voicemail auf sein Handy. Bei der Anfahrt zum Flughafen informiert ihn das GPS über freie Parkplätze im Parkhaus des Flughafens und führt ihn zu diesen. Das Roadpricing-System verrechnet automatisch die Parkgebühr. Der automatische Check-in des Flughafens registriert die Ankunft des Reisenden aufgrund des Eintritts des Handys in den Flughafenbereich. Eine SMS-Nachricht teilt ihm mit, an welchem Gate er einsteigen kann und welchen Sitz er erhalten hat. Beim Boarding identifiziert sich der Passagier über seine Kundenkarte (Smart Card). Da sein Handy und sein PC an Board über einen Internetzugang verfügen, erlauben sie ihm an Board ein Arbeiten wie im Büro.

Grosse Teile dieses Szenarios sind bei Fluggesellschaften bereits in Vorbereitung. Ähnliche Szenarien gibt es für die Pflege von Patienten zu Hause, für Servicemitarbeiter in der Maschinenwartung, für die Unterhaltung mit Audio, Video und Spielen und weitere Bereiche. Die Anwendungsgebiete für CSAs liegen primär in all jenen Bereichen des geschäftlichen und privaten Lebens, die von der derzeitigen Vernetzung durch lokale Netze und das Internet noch nicht erreicht werden, d.h. vor allem im mobilen Einsatz und in Privathaushalten. Dort ermöglichen sie

1 Eine weitere Ausdifferenzierung der Connected Smart Appliances in Connected Smart Appliances im engeren Sinne (=neue kleine Geräte mit dem primären Zweck in Kommunikation, z.B. PDAs, Mobiltelefone und Chipkarten) und Connected Smart Assets (=intelligente Dinge, bei denen die Informationsverarbeitung unsichtbar ist) findet sich bei [Fleisch, 2001 \#781]. 
eine 'Immediacy of Data', d.h. die Beschleunigung und Verkürzung verschiedenster Prozesse durch die Eliminierung umständlicher Tätigkeiten zur Datenerfassung und -zugriff [vgl. Österle 2000].

Forschung und Praxis sind von einer gemeinsamen Vision der Potentiale und einer einheitlichen Terminologie der CSAs noch weit entfernt. Dieses Kapitel liefert einen Überblick über den aktuellen Stand der Entwicklung von CSAs bzgl. der zugrundeliegenden Technologien und ihrer betriebswirtschaftlichen Potentiale.

\subsection{Fallbeispiele}

\subsubsection{Safeway}

Die britische Supermarktkette Safeway hat in Zusammenarbeit mit IBM das sog. 'Easi Order'-System entwickelt, das Kunden zur Verfügung gestellt wird, die Inhaber einer Customer Loyalty Card sind. Easi Order basiert auf einem modifizierten Palmpilot, der mit einem zusätzlichen Barcode-Leser ausgestattet ist.

Mit dem Gerät können Kunden zu Hause oder im Büro Einkaufslisten zusammenstellen, indem Produkte manuell eingegeben oder über deren Barcode erfaßt werden. Die fertige Liste wird anschließend über das Internet an den nächsten Safeway Store übertragen. Der Kunde hat die Möglichkeit, die genaue Zeit vorzugeben, zu der er die Waren abholen möchte. Bei seiner Ankunft sind die Waren bereits fertig verpackt und können ohne Wartezeit bezahlt werden.

Durch das System erwartet Safeway eine Zeitersparnis von 60-90 Minuten pro Woche für den durchschnittlichen Kunden. Erreicht wird dies u.a. durch spezielle Easi Pay-Stationen, die die elektronischen Einkaufslisten direkt weiterverarbeiten können. Darüber hinaus erhält der Kunde über das Gerät Einkaufsvorschläge, Rezepte und vorgefertigte Einkaufslisten, die auf Basis früherer Einkäufe zusammengestellt werden und den Einkauf noch weiter vereinfachen sollen.

Safeway erhält durch das System neben der verstärkten Kundenbindung wertvolle Informationen über Einkaufstrends und Kundenprofile. Dies ermöglicht eine stetige Verbesserung des eigenen Sortiments und individualisierten Sonderangeboten. Das Unternehmen plant, Easi Order schrittweise in allen Märkten anzubieten und auch auf andere Endgeräte wie Screenphones oder Internet-TV auszudehnen. 


\subsubsection{U.S. Postal Service}

Der U.S. Postal Service setzt seit Mitte des Jahres 1999 im Rahmen des Pilotprojekts 'Surface 2000' sog. 'Smart Labels' im Paketversand ein. Die zugrundeliegende Technologie liefert die Firma Motorola und ermöglicht eine 'Radio Frequency Identification (RFID)', d.h. eine Identifikation von Gegenständen durch drahtlose Datenübertragung.

Smart Labels werden wie herkömmliche Paketaufkleber verwendet und sind mit einem Barcode bedruckt, der mit einem normalen Barcodeleser verarbeitet werden kann. Darüber hinaus befindet sich auf der Rückseite der Smart Labels aber auch ein Transponder, d.h. ein Halbleiter, der ohne eigene Stromversorgung auskommt und von außen über elektromagnetische Felder mit der notwendigen Energie versorgt wird. Über entsprechende Sendestationen, die z.B. in einem Postwagen angebracht sein können, ist es damit möglich, alle Informationen über das Paket aus dem Smart Label abzurufen, die normalerweise manuell erfaßt werden müßten.

Smart Labels erlauben dem Paketversender eine automatisches Verfolgen von Paketen beim Ein- und Ausladen aus dem Lieferwagen. Einerseits ist so der aktuelle Aufenthaltsort eines Pakets jederzeit nachvollziehbar, andererseits wird die Zahl fehlgeleiteter oder verlorener Pakete stark reduziert. Darüber hinaus wird die Automatisierung der Paketlogistik deutlich vereinfacht. Da mit Smart Labels auch weiterhin die herkömmlichen Barcodes eingesetzt werden können, ist eine fließende Migration zum neuen System machbar.

\subsubsection{Siemens HomeAssistant}

Der HomeAssistant von Siemens ist eine Steuersoftware für Haushaltsgeräte, die bereits mehrfach in privaten Häusern installiert wurde. An mehreren Stellen im Haus können Touchscreens ausgestellt werden, die den Zugriff auf alle Funktionen des Systems erlauben. Über seine Steuerungsfunktionen hinaus dient der HomeAssistant gleichzeitig zum Telefonieren, Faxen, Musik hören, Fernsehen, Spielen, als Homebanking-Terminal, Gesundheitsratgeber, hausinterne Notrufsäule usw. (siehe Bild 12-1). 


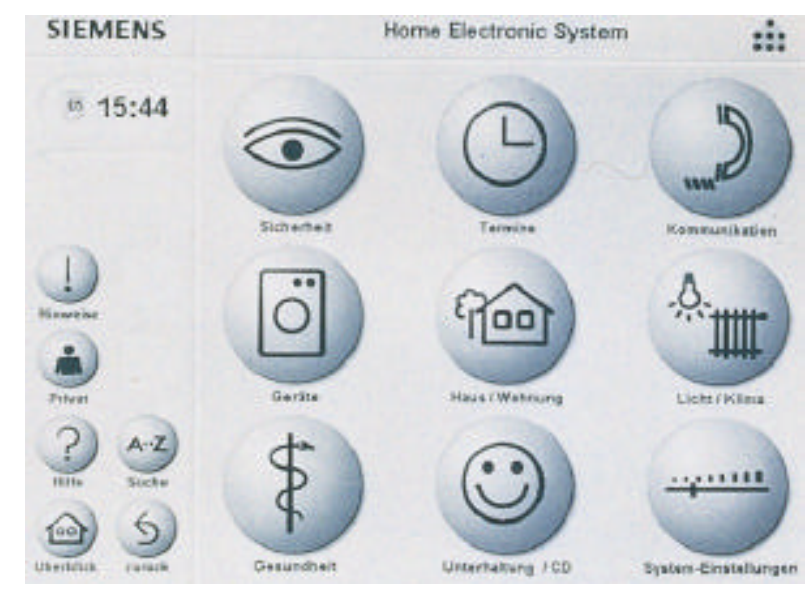

Bild 12-1: HomeAssistant ${ }^{\circledR}$ von Siemens

Die Anwendung 'Überwachung' gibt den Hausbewohnern auf Knopfdruck einen Überblick über den Zustand ihres Hauses bzw. ihrer Wohnung und der sich dort befindlichen Geräte. Vom Übersichtsknopf in der Startmaske des HomeAssistant gelangt der Benutzer direkt in die Maske 'Überwachungsfunktionen'. Dort findet er Zusatzinformationen wie 'Fenster offen', 'Flurlicht brennt', 'Bewegungsmelder aktiv', aber auch Fehlermeldungen wie 'Heizung ausgefallen'. Kritische Meldungen werden zusätzlich über Alarme, wie Warnton oder Blinken, am HomeAssistant gemeldet. Die Zustandsinformationen können sowohl am HomeAssistant $\mathrm{zu}$ Hause als auch von unterwegs über das Telefon abgefragt werden. Ebenso ist es möglich, die Überwachungsfunktion vor Ort oder über das Telefon zu aktivieren bzw. zu deaktivieren.

\subsection{Einsatzbereiche von CSAs}

Aufgrund des vielfältigen Erscheinungsbilds und der Vielzahl möglicher Funktionalitäten ist der Einsatz von CSAs nicht auf einige wenige Prozesse beschränkt. Das Anwendungsgebiet umfaßt prinzipiell alle Aufgaben des privaten und betrieblichen Alltags, in denen Aktivitäten zum Datenzugriff oder der Datenerfassung enthalten sind (siehe Bild 12-2). Dies gilt insbesondere für den mobilen Einsatz, in dem herkömmliche Informationstechnologie nur beschränkt verwendbar ist. 


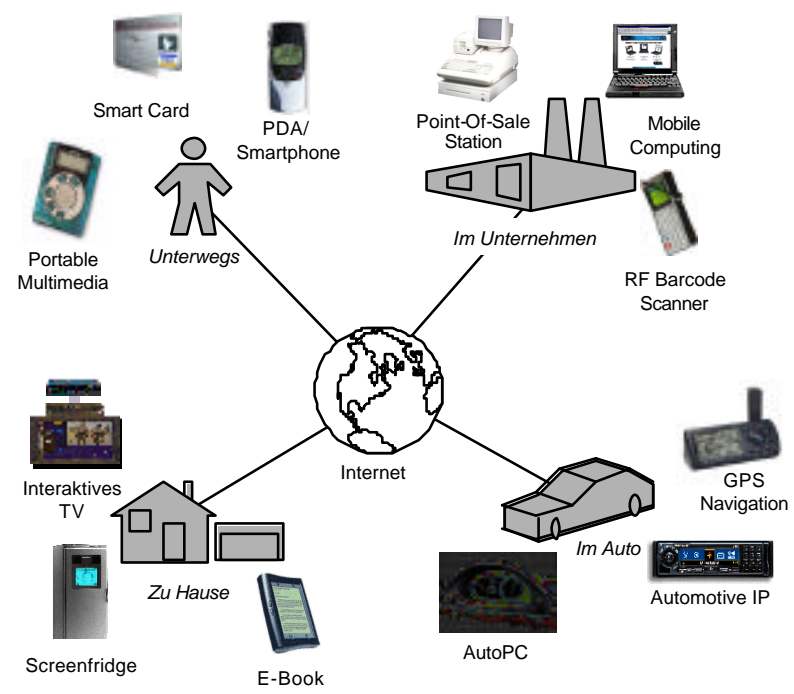

Bild 12-2: Beispiele für Einsatzbereiche von CSA-Technologien

Die Anwendung von CSAs läßt sich je nach Einsatzbereich wie folgt klassifizieren [vgl. Powers 1999]:

- $\quad$ Privatleben (Business-to-Consumer (B2C))

'Intelligente' Haushaltsgeräte unterstützen den Konsumenten bei zahlreichen Aktivitäten der Haushaltsführung, beim Einkaufen, auf Reisen usw. Externe Dienstleister übernehmen die Wartung von Haushaltsgeräten oder den Schutz vor Einbrechern. Darüber hinaus machen Unterhaltungsangebote aus dem Netz (Multiplayer-Spiele, Video-on-Demand) dem traditionellen Fernsehangebot Konkurrenz. Stichworte sind hier Home of the Future, Entertainment off the Net und Automotive IP.

- Unternehmen (Business-to-Business (B2B))

Im betrieblichen Umfeld sind CSAs vor allem für den mobilen Einsatz relevant, d.h. in den Prozessen des Vertriebs, Verkaufs oder des Kundendiensts. Darüber hinaus können aber auch viele Datenerfassungsaufgaben innerhalb eines Unternehmens automatisiert werden, z.B. in der Produktion oder der Logistik. Stichworte sind hier Personal Information Management, Field Force Automation, Shop Floor Control sowie Factory and Warehouse Automation.

\subsection{Technologien von CSAs}

Bei den Geräten ist sowohl ein Trend zur Integration von Funktionalitäten als auch zur Spezialisierung zu beobachten (vgl. [Burrows/Reinhardt 1999], [Kuri 1999]). 
Auf der einen Seite implementieren beispielsweise die Hersteller von Mobiltelefonen immer mehr Funktionen wie Terminkalender, eMail, SMS-Nachrichten, Adreßdatenbank, Internetbrowser, Remote Control für beliebige Geräte und Videokommunikation in ihre Produkte mit dem Ziel eines universellen PDAs (Personal Digital Assistant). Auf der anderen Seite erhalten Spielgeräte, Fernseher, Kühlschränke, Heizungsanlagen, Maschinensteuerungen zunehmend eigene, spezialisierte Prozessoren, Netzanschlüsse und Bedienungseinheiten.

Gleichzeitig ist der Kampf um die einheitliche Bedienungsoberfläche zwischen den Betriebssystemherstellern in vollem Gange: Windows CE vs. Java vs. EPOC. Noch entscheidender für die Vernetzung der intelligenten Geräte ist der Wettbewerb von Middleware wie UPnP, Jini oder WAP sowie alternativer Netzwerke. Die wichtigsten Varianten sind hier spezielle Verkabelungen (z.B. IEEE1394, USB), Telefonleitung (z.B. HomePNA), Stromleitung (z.B. CEBus, X-10), Funk im Nahbereich (z.B. HomeRF, Bluetooth, IrDA) und Funk im Fernbereich (z.B. GSM, UMTS). Am Ende wird sich voraussichtlich eine Kombination von Technologien mit jeweils einem marktbeherrschenden Standard durchsetzen. Wegen ihrer riesigen Volumina wird die Technologie der Konsumentenelektronik auch die Standards für die Unternehmen liefern, wie dies bei den Personal Computern schon der Fall war.

Die zur Realisierung von CSAs notwendigen Informationstechnologien lassen sich in folgende vier Gruppen einteilen (siehe auch Bild 12-3):

- Anwendungsplattform

Die Anwendungsplattform entspricht dem Betriebssystem eines konventionellen Informationssystems. Sie stellt Funktionalitäten für den Zugriff auf die Hardware der CSAs und das angeschlossene Netzwerk zur Verfügung. Da CSA-Anwendungen häufig auf unterschiedlicher Hardware (z.B. variierende Displaygrößen, Benutzereingabe durch Touchscreen, Sprache usw.) arbeiten müssen, besteht eine der wichtigsten Eigenschaften der Anwendungsplattform in der Bereitstellung einer hardwareunabhängigen Schnittstelle, auf die die Anwendung zurückgreifen kann.

- $\quad$ Middleware

Die Middleware einer CSA dient als logische Schnittstelle zu anderen CSAs und ermöglicht den Datenaustausch bzw. die gegenseitige Nutzung der Funktionalität von CSAs. Die CSA-Middleware liefert einen Überblick über die im jeweiligen Netz vorhandenen CSAs und die von ihnen angebotenen Funktionen. Darüber hinaus regelt sie die Kommunikation zwischen CSAs über klar definierte Schnittstellen.

- Netzwerkinfrastruktur

Die Netzwerkinfrastruktur stellt die physische Basis dar, auf der sich ein beliebiger Datenaustausch zwischen mehreren CSAs abspielt. Wie in konventionellen Rechnernetzen auch, kommen hier elektrische, optische sowie drahtlose Übertragungsverfahren zum Einsatz. Im Gegensatz zu unternehmensin- 
ternen Netzen werden bei CSAs vor allem Verfahren favorisiert, die bereits bestehende Verkabelungen nutzen, wie z.B. Stromleitungen, oder die drahtlos arbeiten.

- $\quad$ Schnittstelle zu eServices

Eine weitere Middleware ermöglicht die Kommunikation von CSAs mit Anwendungen außerhalb des jeweiligen Netzwerks, insbesondere des Internets. Beispiele dafür sind u.a. Energiemanagement- oder Überwachungsdienste für Wohnhäuser oder Application Services im Internet.

Die Basis für den Einsatz all dieser Technologien ist die Adressierbarkeit von CSAs, d.h. um die Vernetzung zu ermöglichen benötigt jede CSA eine eigene IPAdresse. Die Etablierung eines Protokollstandards wie IPv6, der den bisherigen IP-Adressraum erweitert, ist daher die wichtigste Voraussetzung für die Realisierung von CSAs.

Neben den genannten Standards kommen in CSAs auch eine Vielzahl anderer Technologien zum Einsatz wie z.B. biometrische Verfahren zur Erkennung von Gesichtern und Fingerabdrücken sowie Sprachverarbeitung oder Transpondertechnologie zur Herstellung extrem flacher Transistoren, die keine eigene Stro mversorgung benötigen.

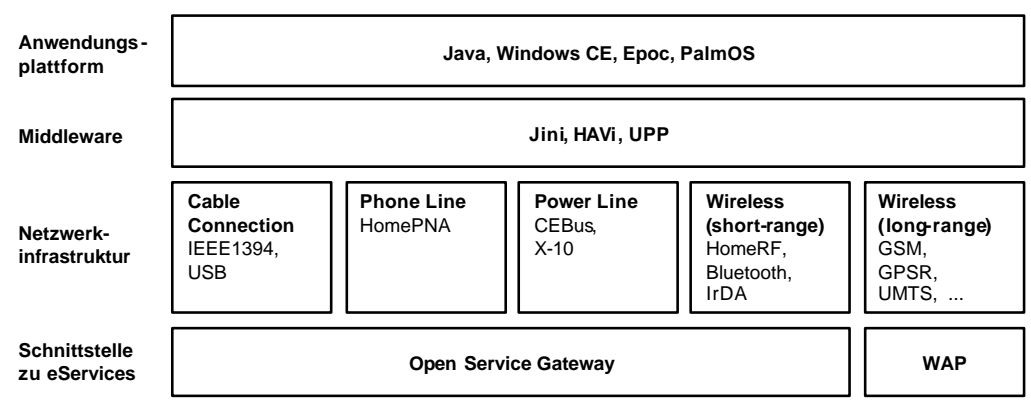

Bild 12-3: Basistechnologien für CSAs

\subsection{Treiber der CSAs}

Nahezu alle Anbieter von Hard-/Software, Netzwerkinfrastruktur und Inhalten (Unterhaltung, Wirtschaftsinformationen, usw.) investieren mittlerweile in die Entwicklung von CSAs. Die Vorreiter dieser Entwicklung sind internetfähige Fernseher, Screenphones und Personal Digital Assistants [vgl. Miller 1999]. Ein typisches Beispiel für CSAs, die bereits heute enorm hohe Verkaufszahlen erre ichen, ist die Spielkonsole Dreamcast der Firma Sega. Neben herkömmlichen Vi- 
deospielen erweitert das Gerät den heimischen Fernseher um einen Internetbrowser und erlaubt die Teilnahme an Multiplayer-Spielen über das Internet.

Dem Markt für Heimvernetzung werden hohe Wachstumsraten für die kommenden Jahre vorausgesagt. Während bis 2001 die eingesetzten Technologien noch aus der konventionellen PC-Technologie stammen und auf meist statische Inhalte zugreifen werden, wird sich nach und nach die Vernetzung beliebiger Haushaltsgeräte durchsetzen, die mittels Breitbandanschlüssen an hochwertige Dienste angebunden werden (siehe Bild 12-4).

Der Markt für mobile Endgeräte wird derzeit vor allem von der Verbreitung Internet-fähiger Mobiltelefone vorangetrieben. Bis zum Jahr 2003 wird beispielsweise erwartet, daß ein Drittel der europäischen Bevölkerung ein WAP-fähiges Handy besitzt und nutzt. Dieses erlaubt dann den Zugang zu nahezu beliebigen Anwendungen bzw. Diensten im Netz.

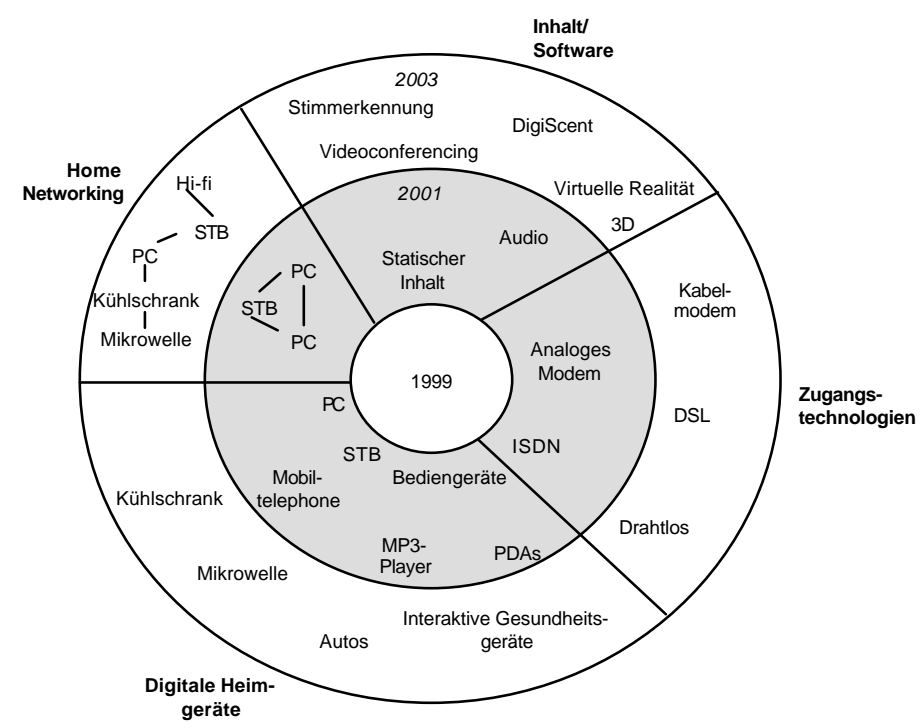

Bild 12-4: Ausstattung vernetzter Haushalte [vgl. Watamonitor, 2000 \#2415]

Folgende Entwicklungen unterstützen den CSA-Trend über die nächsten Jahre: ${ }^{2}$

2 Vgl. dazu [Boriello/Want, 2000], [Gershenfeld 1999], [Hansmann et al. 2001], [Jahnke 1998], [Mattern 2000], [Mattern 2000], [Mattern 2001], [Norman 1998], [Tennenhouse 2000], [Weiser 1991], [Weiser 1993] und [Weiser/Brown, 1995] 
- Bei gleichzeitig steigender Leistungsfähigkeit fallen die Herstellungskosten weiter.

Die Leistung von Mikroprozessoren verdoppelt sich alle achtzehn Monate wobei sich deren Preise im gleichen Zeitraum halbieren. Nahezu seit ihrer Geburtsstunde im Jahr 1971 folgen die Mikroprozessoren dem Gesetz der parallelen Preisdegression und Leistungsprogression, welches Gordon Moore 1975 im "Moor'schen Law" erstmals formulierte (vgl. [Kelly 1998, S. 52], [Gilder 1997]). Dieses Gesetz fasst auch heute die Preis-/Leistungsentwicklung nach wie vor zutreffend zusammen. Die Kosten für den Einsatz von Mikroprozessortechnologien in den verschiedensten Gebrauchsgegenständen können bzgl. des Preis -/Leistungsverhältnisses mit konventionellen Verfahren wie z.B. Barcodes ohne weiteres konkurrieren.

- Das Preis-/Leistungsverhältnis der Kommunikation steigt.

Die zur Verfügung stehende Bandbreite in Fest- und Mobilnetzen wird durch innovative Verfahren (z.B. zur Datenkompression) effizient genutzt, so daß auch ressourcenintensive Angebote wie z.B. Video-on-Demand möglich werden. Nach der Regel von Gilder verdreifacht sich die Bandbreite von Kommunikationsnetzwerken in den nächsten 25 Jahren alle zwölf Monate und der Preis pro übertragenes Bit konvergiert gegen Null. "In essence, as frequencies rise and wavelengths drop, digital performance improves exponentially. Bandwidth rises, power usage sinks, antenna size shrinks, interference collapses, error rates plummet" [Gilder 1997].

- Mit zunehmendem Reifegrad sinkt die Mindestgröße von Technologien.

Dies gilt ebenso für Computer wie für Verbrennungs- oder Elektromotoren sowie Uhrwerke. [Kelly 1998, S. 19] sagt aus: "As technology becomes ubiquitous it also becomes invisible", und bringt damit zum Ausdruck, daß sich die Informatisierung erst dann durchgesetzt hat, wenn Computer nicht mehr als solche zu erkennen sind und Teil nahezu aller Objekte des täglichen Lebens geworden sind [vgl. Gershenfeld 1999]. Ein Beispiel in der IT sind 'Embedded Networked Processors', sog. 'Jelly Beans'. Jelly Bean ist ein Samme 1begriff für sehr kleine, kommunikationsfähige und typischerweise sehr einfach strukturierte Informations- und Kommunikationstechnologie, die in Zukunft in Massen produziert wird und deren Stückkosten gegen Null gehen werden (vgl. [Davis/Meyer 1998a], [\Kelly, 1998 \#602, S. 11ff.]).

- Der Energieverbrauch von Mikroprozessoren sinkt.

Technologien zur Energieversorgung von Mikroprozessoren entwickeln sich laufend weiter.

- Permanenter Netzzugang wird auch für Privatpersonen erschwinglich.

Konsumenten und Mitarbeiter haben ohne Verzögerung real-time und überall Zugriff auf alle privaten und geschäftlichen Informationen. Sie benutzen dazu das jeweils geeignetste Gerät ('Multi-mode Access'). 
- Unterscheidung zwischen Festnetz und Mobilfunk wird irrelevant.

CSAs erfassen die Daten direkt am Ort des Geschehens, wo auch immer dies ist. Smart Cards und Point-of-Sales-Kassen erfassen den Zahlungsvorgang, die gekauften Artikel, die Uhrzeit und den Ort. Die Bank, das Kaufhaus und die Lieferanten des Kaufhauses haben real-time Zugang zu diesen Daten.

- $\quad$ Standards breiten sich in Reichweite und Inhalt aus.

Wie in zahlreichen anderen Fachgebieten sind Standards auch in der Informationsverarbeitung eine Voraussetzung zur Nutzung der Effekte der Economies of Scale bei gleichzeitiger Individualisierung von Leistungen. Aufwärtskompatible bzw. erweiterbare Informations- und Kommunikationsstandards mit breiter Akzeptanz sind Grundlage der Informatisierung der Wirtschaft. Der eigentliche Wert des Internets liegt in der Schaffung eines nahezu weltweit akzeptierten Kommunikationsstandards (TCP/IP) und eines Standards zur Präsentation von Dokumenten (HTML) [Malkin 1995]. Über das Internet könnte jeder Computer oder jedes CSA Zugriff zu allen Informationen bekommen, die irgendein anderer Computer oder ein anderes CSA auf dem Netz hält. Das Navigationssystem GPS beispielsweise hat also Zugriff auf Straßenkarten, Staumeldungen, Wetterberichte, Hotels, Restaurants, KfzWerkstätten, usw.

- Technologien zur Sprach- und Texterkennung sind ausgereift.

Die Benutzerfreundlichkeit von Informationssystemen hat sich weiter verbessert. Automatische Erkennung von Sprache und Schrift hat in vielen Bere ichen die manuelle Eingabe ersetzt.

- Einheitliche, dauerhafte Netzadressen für jedermann und jedes Ding.

Jedes Gerät resp. jede Person erhält eine eindeutige und langfristige Identifikationsnummer und wird so zum dauerhaften Bestandteil des globalen Informationsuniversums.

- Anbieter offerieren eine breite Palette von Inhalten und Anwendungen. Es etabliert sich ein umfassendes Angebot an Diensten auf dem Netz, das für Privatpers onen und Unternehmen einen attraktiven Nutzen bietet.

\subsection{Betriebswirtschaftliche Effekte von CSAs}

\subsubsection{Neue Prozesse durch Automatisierung der Datenerfassung}

CSAs automatisieren die Dateneingabe. Sie verhindern damit Medienbrüche und führen zu neuen inner- und überbetrieblichen Prozessen. Wie oben bereits angedeutet konzentrieren sich Forschung und Praxis heute primär auf die Vernetzung 
von Unternehmen, Prozessen, Informationssystemen und Menschen. Sie verwenden dabei die Vermeidung von Medienbrüchen als zentrales Argument zur Steigerung der Effizienz in Netzwerken. Ein häufig genanntes Beispiel für einen Medienbruch ist die mehrfache Erfassung eines Auftrags in unterschiedliche betriebliche Informationssysteme innerhalb einer Wertschöpfungskette. Ein Medienbruch ist vergleichbar mit einem fehlenden Glied in einer Informationskette und ist Mitursache für Langsamkeit, Intransparenz, Fehleranfälligkeit, etc. inner- und überbetrieblicher Prozesse.

Die oben erwähnte neue Vernetzungsebene adressiert das zur Zeit größte Problem der Informationsverarbeitung: den Medienbruch Dateneingabe. Ihr Ziel ist es, die physische Welt (Menschen, Produkte, Betriebsmittel, etc.) mit der Informationssystemwelt (z.B. ERP-, EC-, und SCP-Systeme sowie lokale, regionale und globale Informationsnetzwerke) zeitnah und kostengünstig zu vernetzen und damit die Lücke zwischen der physischen betrieblichen Realität und deren informationstechnologischem Abbild zu schließen (siehe Bild 12-5). Dies gelingt erst dann, wenn es den Menschen als Mediator zwischen physischer und informatischer Welt nicht mehr braucht, d.h. wenn physische Ressourcen ohne menschliche Intervention automatisch mit den Rechnernetzwerken (oder unter sich) kommunizieren.

Mit den heute in der Praxis eingesetzten Technologien zur Vernetzung von physischen Ressourcen mit Informationssystemen wie z.B. der Dateneingabe von Hand über die Tastatur, die Spracheingabe oder das Scannen von Barcodes, ist dies noch nicht möglich. Aktuelle Entwicklungen im Bereich von passiven und aktiven Tags, die auf der Radio Frequency Identification (RFID)-Technologie aufbauen, zeigen jedoch einen möglichen Entwicklungspfad auf. Sie führen zu neuen Szenarien, in welchen Unternehmen ihre 'Assets' mit (a) Intelligenz ausstatten ('smart' machen), indem diese z.B. ihren Ziellagerort kennen oder laufend ihre Umgebungstemperatur aufzeichnen, und (b) diese 'Assets' automatisch mit den hausinternen Informationssystemen verknüpfen ('connected'). Diese CSAs ermöglichen eine neue Qualität an zentral und dezentral gesteuerten Prozessen, die von der dynamischen Preisbildung für einen Salatkopf (nach der Differenz zwischen der aktuellen Zeit und dem Erntezeitpunkt, der Temperatur in der Auslage und der Qualität der um den Verkauf konkurrierenden anderen Salatköpfe) bis zur innerund überbetriebliche Logistik reichen. 


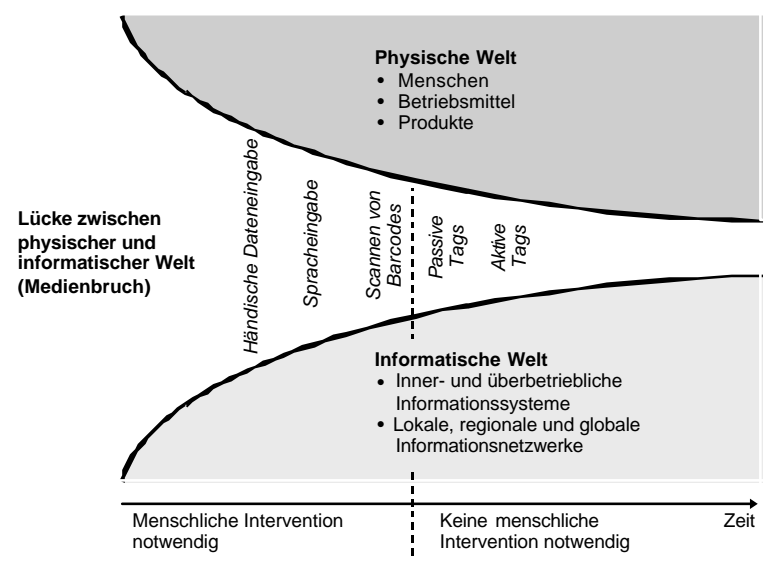

Bild 12-5: Lücke zwischen physischer und informatischer Welt ${ }^{3}$

\subsubsection{Management von komplexen Systemen durch Verteilung von Wissen und Intelligenz}

CSAs sind hybride Produkte, die sich aus physischen Leistungen und Informationsleistungen zusammensetzen. Sie besitzen neben den klassischen Dimensionen von Produkten auch Dimensionen der Datenverarbeitung und sind Empfänger, Sender, Träger, Sammler und Verarbeiter von Daten. Diese Dimensionen geben CSAs neue Eigenschaften (siehe Tabelle 12-1). Beispielsweise können CSAs:

- Wissen, z.B. über sich selbst, lokal, d.h. direkt in sich selber, speichern,

- das Wissen berührungslos und global, d.h. z.B. einer Gemeinschaft von weiteren CSAs oder einem Rechnernetzwerk wie dem Internet, zur Verfügung stellen,

- globales Wissen empfangen,

- $\quad$ selbständig Umgebungswissen wie z.B. Temperatur, Erschütterung, Wassergehalt sammeln und

- $\quad$ schließlich all dieses Wissen lokal vera rbeiten.

CSAs eignen sich damit zur Verteilung von Wissen bzw. Intelligenz an den Ort, an dem das Wissen verwendet bzw. erzeugt wird. Dies kann beispielsweise das lokale CSA sein, eine lokale Gruppe von CSAs oder ein globales Informationssystem. Den Erkenntnissen der Komplexitätstheorie folgend ('Distribute Being',

${ }^{3}$ Gemeinsam mit Intellion AG entwickelt 
'Control from Bottom-up' [Kelly 1995]) öffnen CSAs damit eine neue Tür zur Steuerung komplexer Systeme.

\begin{tabular}{|l|l|}
\hline \multicolumn{1}{|c|}{ CSA-Dimension } & \multicolumn{1}{c|}{ CSA-Eigenschaft } \\
\hline Datenempfänger & $\begin{array}{l}\text { Automatisches berührungsloses Aufnehmen } \\
\text { von 'globalem' Wissen }\end{array}$ \\
\hline Datensender & $\begin{array}{l}\text { Automatisches berührungsloses Übermitteln } \\
\text { von lokalem Wissen }\end{array}$ \\
\hline Datenträger & $\begin{array}{l}\text { Lokales Wissen über 'Asset' immer bei } \\
\text { 'Asset' }\end{array}$ \\
\hline Datensammler & $\begin{array}{l}\text { Automatische lokale Wissenserweiterung, } \\
\text { wie z.B. Temperaturaufzeichnung }\end{array}$ \\
\hline Datenverarbeiter & Lokale Wissensverarbeitung \\
\hline
\end{tabular}

Tabelle 12-1: Verteilte Intelligenz durch CSAs

\subsection{Unternehmerische Konsequenzen}

Die Konsequenzen von CSas sind noch schwer abschätzbar. Wer hätte z.B. vor drei Jahren erwartet, daß das vom Fraunhofer-Institut in Erlangen entwickelte MP3-Format, das die komprimierte, digitale Speicherung von Musik erlaubt, zlsammen mit dem Internet innerhalb weniger Jahre die gesamte Musikbranche restrukturieren würde [vgl. Gomes 1999]. Dennoch lassen sich einige Herausforderungen jetzt schon feststellen:

- Geräte- und Softwarehersteller

Die größte Herausforderung betrifft die Hersteller der Geräte und der Software. Das reicht von der elektronisch überwachten Etikettendruckmaschine über die Rolltreppe bis zur Büro-Kaffeemaschine, die den Kaffeeverbrauch für die Nachbevorratung an die Servicefirma weiterleitet. MP3 und andere Multimedia-Standards bringen eine neue Generation von Geräten zur Audio/Video-Speicherung und -wiedergabe hervor.

- Dienstleistungsanbieter

Vernetzte intelligente Geräte sind gewöhnlich nur zusammen mit einem speziellen Service sinnvoll. Im Reisebeispiel ist dies der Check-in und das Boarding, bei LifeShirt die Datenauswertung, bei MP3 der Musikverteilungsservice und bei einer Spielkonsole wie Dreamcast die Multiplayer-Spiele. 
- Anbieter klassischer Produkte und Dienstleistungen

Intelligente Geräte verändern das Business an vielen Stellen. Unternehmer müssen sich die Frage stellen: Welche Informationen und elektronischen Dienstleistungen schaffen den Konsumenten, Mitarbeitern oder Maschinen am Ort des Geschehens Zusatznutzen (z.B. ein persönliches Telefonverzeichnis auf dem Netz, eine regionale Wettervorhersage über das Handy)? Welche Informationen können intelligente Geräte vom Ort des Geschehens liefern (z.B. Abverkaufsdaten von Point-of-Sales-Kassen)? Welche Marktteilnehmer sind in der Lage, unsere Produkte und Dienstleistungen besser oder billiger anzubieten (z.B. Wirtschaftsnachrichten aus Sicht einer Tageszeitung)?

Obwohl diese Technologie - verglichen etwa zu ERP-Systemen - noch sehr unreif ist, läßt sie gravierende Veränderungen in Geschäftsprozessen und im privaten Bereich erahnen. Dies löst - zumindest in einigen Ländern - massive Ängste vor der Technisierung und der Überwachung aus. CSA-Anwendungsbeispiele mit einer schwerwiegenden gesellschaftspolitischen Dimension sind etwa das Monitoring von gebrechlichen oder geistig verwirten Mitmenschen, von Kindern oder von Sträflingen. Hier vermischen sich Fragen der Ethik mit jenen der Datensicherheit und verlangen nach einer Diskussion, in welcher der technologische und damit betriebswirtschaftliche Fortschritt eine sekundäre Rolle einnimmt.

[Boriello/Want, 2000], Boriello, G., Want, R., Embedded Computation meets the World Wide Web, in: Communications of the ACM, May 2000 / Vol. 43 No. 5, S. 59-66

[Gershenfeld 1999] Gershenfeld, N., Wenn die Dinge denken lernen, Econ, München, Düsseldorf, 1999

[Hansmann et al. 2001] Hansmann, U., Merk, L., Nicklous, M.S., Stober, Th., Pervasive Computing Handbook, Spinger, Berlin etc. 2001

[Jahnke 1998], Jahnke, A., Tomorrowland, CIO Web Business Magazine, 12/1998, http://www.cio.com/archive/webbusiness/120198_qa.html

[Mattern 2000], Mattern, F, Ubiquitous Computing, Unterlagen zur Vorlesung am Departement Informatik an der ETHZ, 2000

[Mattern 2000], Mattern, F., State of the Art and Future Trends in Distributed Systems and Ubiquitous Compting, Working Paper, Departement of Computer Science, ЕГН Zurich, http://www.inf.ethz.ch/vs/publ/papers/DisSysUbiCompReport.pdf

[Mattern 2001], Mattern, F., Das aktuelle Schlagwort: Pervasive Computing / Ubiquitous Computing, , Working Paper, Departement of Computer Science, ETH Zurich, http://www.inf.ethz.ch/vs/publ/papers/PvCubi.pdf 
[Norman 1998] Norman, D.A., The Invisible Computer, MIT Press, Cambridge, London 1998

[Tennenhouse 2000], Tennenhouse, D., Proactive Computing, in: Communications of the ACM, May 2000 / Vol. 43 No. 5, S. 43-50

[Weiser 1991] Weiser, M., The Computer of the $21^{\text {st }}$ Century, in: Scientific American, September 1991

[Weiser 1993] Weiser, M., Some Computer Science Issues in Ubiquitous Computing, Communications of the ACM, July 1993

[Weiser/Brown, 1995] Weiser, M., Brown, J.S., The Coming Age of Calm Technology, Xerox PARC, October 5, 1995

[Fleisch 2001] Fleisch, E., Ubiquitous Computing in der Logistik: Anwendungsbeispiel und betriebswirtschaftliche Auswirkungen, eingereicht in: Wirtschaftsinformatik 2001, Augsburg 2001 